\title{
On the Mathematical Basis of the \\ Linear Sampling Method
}

\author{
F. Cakoni \\ Department of Mathematical Sciences \\ University of Delaware \\ Newark, DE 19716 \\ D. Colton \\ Department of Mathematical Sciences \\ University of Delaware \\ Newark, DE 19716
}

Technical Report No. 2002-1

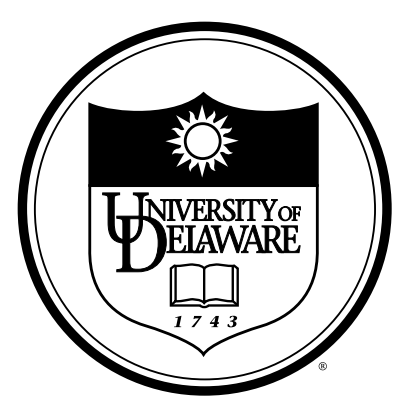

DEPARTMENT

$\mathrm{OF}$

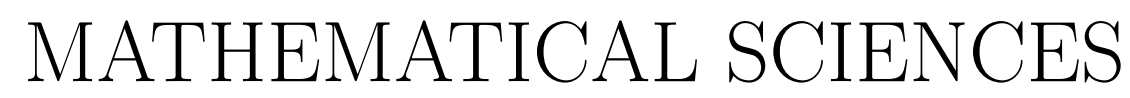

University of Delaware

Newark, Delaware 


\title{
ON THE MATHEMATICAL BASIS OF THE LINEAR SAMPLING METHOD
}

\author{
FIORALBA CAKONI and DAVID COLTON \\ Department of Mathematical Sciences \\ University of Delaware \\ Newark, Delaware 19716
}

\begin{abstract}
The linear sampling method is an algorithm for solving the inverse scattering problem for acoustic and electromagnetic waves. There are two versions of the linear sampling method. Although the second version is on a firm mathematical foundation, it is restricted to the case of non-absorbing media, full aperture scattering data or, more specifically, to the cases when the far field operator is normal. The first version of the linear sampling method is more flexible, being able to treat the aforementioned cases as well as partially coated obstacles. However, its mathematical foundation is less well established. In this paper we provide arguments giving a mathematical justification of the first version of the linear sampling method.
\end{abstract}

Keywords: inverse scattering, mixed boundary conditions, linear sampling method, inhomogeneous media.

\section{Introduction}

The inverse scattering problem of interest to us in this paper is that of determining the shape of a two dimensional scattering object from a knowledge of the far field pattern of the scattered wave where the incident field is a time harmonic plane wave. (The restriction to two dimensions is purely for convenience and all our results remain valid in the three dimensional case). Until recently, the solution of 
this problem required more information, in particular whether or not the scattering object was penetrable or impenetrable as well as what boundary conditions were satisfied by the scattered field on the boundary of the scatterer $D$. However, in 1996 a method was introduced which does not require this extra information and in addition is a linear algorithm for the determination of the boundary $\Gamma$ of the scatterer [4]. This method is called the linear sampling method and has been the subject of considerable attention since its introduction [2], [10]-[12].

There are two versions of the linear sampling method. To describe these two methods we assume the incident field $u^{i}$ is given by (factoring out a term of the form $e^{-i \omega t}$ where $\omega$ is the frequency and $t$ is time)

$$
u^{i}(x)=e^{i k x \cdot d}
$$

where $k>0$ is the wave number, $\mathrm{d}$ is a unit vector and $x \in \mathbb{R}^{2}$. Then the scattered field $u^{s}(x)=u^{s}(x, d)$ has the asymptotic behavior

$$
u^{s}(x)=\frac{e^{i k r}}{\sqrt{r}} u_{\infty}(\hat{x}, d)+O\left(r^{-3 / 2}\right),
$$

where $u_{\infty}$ is the far field pattern [5]. The first version of the linear sampling method picked a parameter $z \in \mathbb{R}^{2}$ and then looked for a solution $g \in L^{2}(\Omega$ ) (where $\Omega$ is the unit circle in $\mathbb{R}^{2}$ ) of the far field equation

$$
\int_{\Omega} u_{\infty}(\hat{x}, d) g(d) d s(d)=\Phi_{\infty}(\hat{x}, z),
$$

where $\hat{x}=x /|x|$, and $\Phi_{\infty}$ is the far field pattern of the fundamental solution

$$
\Phi(x, z)=H_{0}^{(1)}(k|x-z|)
$$

with $H_{0}^{(1)}$ denoting a Hankel function of the first kind of order zero. It was then shown [3], [4] that for $z \in D$ and almost every $k$ there exists an approximate regularized solution of (1.3) such that

$$
\lim _{z \rightarrow \Gamma}\|g(\cdot, z)\|_{L^{2}(\Omega)}=\infty
$$

and

$$
\lim _{z \rightarrow \Gamma}\left\|v_{g}(\cdot, z)\right\|_{H^{1}(D)}=\infty
$$

where $v_{g}$ is Herglotz wave function with kernel $g$ defined by

$$
v_{g}(x)=\int_{\Omega} e^{i k x \cdot d} g(d) d s(d), \quad x \in \mathbb{R}^{2} .
$$


In particular, $\Gamma$ is characterized by the norm of the regularized solution of (1.3) becoming unbounded. Note that although the proof of this fact required knowing the boundary conditions of $u^{s}$ on $\Gamma$, the equation (1.3) that one needs to solve to determine $\Gamma$ is independent of such knowledge, i.e. $u_{\infty}$ is assumed to be given data (in general noisy).

A major problem with the above approach for solving the inverse scattering problem is that the conclusion (1.5) and (1.6) required that $z \in D$ and nothing was said about what happens if $z \in \mathbb{R}^{2} \backslash \bar{D}$. Since $D$ is unknown this at first glance seems disastrous. However, numerical experiments (c.f. the cover of Vol. 42, number 3 of SIAM Review) indicated that $\|g(\cdot, z)\|_{L^{2}(\Omega)}$ not only became large as $z$ approaches $\Gamma$ from inside $D$ but continued to become larger as $z$ moved into $\mathbb{R}^{2} \backslash \bar{D}$. The fact that this could not be explained mathematically led Andreas Kirsch to introduce a second version of the linear sampling method which is valid for non-absorbing media or, more precisely, when the far field operator $F$ defined by the left hand-side of (1.3) is normal [7], [8]. Under these conditions, Kirsch showed that

$$
\left(F^{*} F\right)^{1 / 4}=\Phi_{\infty}(\cdot, z)
$$

is solvable for almost every $k$ if and only if $z \in D$. Hence if regularization methods are used to solve (1.8), the regularized solution $g=g(\cdot, z)$ has finite norm for $z \in D$ and a "large" norm for $z \in \mathbb{R}^{2} \backslash \bar{D}$ (see again the cover of Vol. 42, number 3 of SIAM Review).

Elegant as Kirsch's result is, it depends on the far field operator being normal. Since this is not a restriction of the first version of the linear sampling method, it is highly desirable to give a mathematical explanation of the observed numerical behaviour of the regularized solution of (1.3) for $z \in \mathbb{R}^{2} \backslash \bar{D}$. This would then put the first version of the linear sampling method on a clearer mathematical foundation. Since only the first version of the linear sampling method is valid for situations involving penetrable absorbing media, limited aperture far field data and partially coated obstacles, this task is particularly important from the point of view of applications. A first attempt to find a solution to this problem was given in [1] for the case of Dirichlet data and smooth boundary $\Gamma$. However the approach in [1] does not readily generalize to more general scattering problems involving Lipschitz boundaries, partially coated obstacles (i.e. mixed boundary conditions) or penetrable absorbing media. The purpose of this paper is to treat these cases together with the simple case of the Dirichlet problem, thus giving a mathematical explanation of the observed numerical behaviour of the first version of the linear sampling method (see the discussion at the end of Section 2.2). This task is accomplished by factoring the far field operator $F$ in the form $F=\mathcal{F} S \mathcal{H}$ where $\mathcal{H}$ maps functions in $L^{2}(\Omega)$ onto Herglotz wave 
functions, $S$ is a solution operator which maps the incident field onto a density function and $\mathcal{F}$ maps the density function onto the far-field pattern. The key fact to note is in the same spirit as Kirsch's result, i.e. $\Phi_{\infty}(\cdot, z)$ is in the range of $\mathcal{F}$ if and only if $z \in D$.

\section{The linear sampling method for the inverse ob- stacle problem}

\subsection{Formulation of the direct and inverse scattering prob- lem}

Let $D \subset \mathbb{R}^{2}$ be an open, bounded region with Lipschitz boundary $\Gamma$ such that $\mathbb{R}^{2} \backslash \bar{D}$ is connected. We assume that the boundary $\Gamma$ has a Lipschitz dissection $\Gamma=\Gamma_{D} \cup \Pi \cup \Gamma_{I}$, where $\Gamma_{D}$ and $\Gamma_{I}$ are disjoint, relatively open subsets of $\Gamma$, having $\Pi$ as their common boundary in $\Gamma$ (see e.g. [9]). Furthermore, boundary conditions of Dirichlet and impedance type with the surface impedance $\lambda \geq 0$ are specified on $\Gamma_{D}$ and $\Gamma_{I}$, respectively. Let $\nu$ denote the unit outward normal vector defined almost everywhere on $\Gamma_{D} \cup \Gamma_{I}$.

We assume that the incident field $u^{i}$ is given by the plane wave $e^{i k x \cdot d}$ where $x \in \mathbb{R}^{2}$, $k>0$ is the wave number and $d$ is a fixed unit vector describing the incident direction. If we denote the scattered field by $u^{s}$ and define the total field by $u(x)=$ $u^{s}(x)+e^{i k x \cdot d}$, then the direct obstacle scattering problem for the obstacle $D$ is to find a weak solution $u \in H_{l o c}^{1}\left(\mathbb{R}^{2} \backslash \bar{D}\right)$ of the following exterior mixed boundary value problem for the Helmholtz equation

$$
\begin{array}{rlll}
\Delta u+k^{2} u & =0 & \text { in } & \mathbb{R}^{2} \backslash \bar{D}, \\
u=0 & \text { on } & \Gamma_{D}, \\
\frac{\partial u}{\partial \nu}+i k \lambda u=0 & \text { on } & \Gamma_{I},
\end{array}
$$

and $u^{s}$ satisfies Sommerfeld radiation condition [5]

$$
\lim _{r \rightarrow \infty} \sqrt{r}\left(\frac{\partial u^{s}}{\partial r}-i k u^{s}\right)=0
$$

where $r=|x|$ and (2.4) is assumed to hold uniformly in $\hat{x}=x /|x|$. In particular the above formulation covers the Dirichlet boundary if $\Gamma_{I}=\emptyset$ and the impedance boundary (Neumann boundary) if $\Gamma_{D}=\emptyset$ and $\lambda>0$ (if $\Gamma_{D}=\emptyset$ and $\lambda=0$ ).

In [1] it was proved that the direct scattering problem (2.1)-(2.4) admits a unique weak solution $u$ and the corresponding scattered field $u^{s}$ has the asymptotic be- 
haviour (1.2) at infinity with the far field pattern $u_{\infty}(\hat{x}, d)$. The inverse obstacle scattering problem is to determine $D$ from a knowledge of $u_{\infty}(\hat{x}, d)$ for $\hat{x}$ and $d$ on the unit circle $\Omega$ and fixed wave number $k$. The far field pattern $u_{\infty}$ defines the far-field operator $F: L^{2}(\Omega) \longrightarrow L^{2}(\Omega)$ by

$$
(F g)(\hat{x}):=\int_{\Omega} u_{\infty}(\hat{x}, d) g(d) d s(d)
$$

Then the linear sampling method looks for a solution $g=g(\cdot, z) \in L^{2}(\Omega)$ of the linear far field equation (1.3) which we write in the form $[2,3,4,7]$

$$
\begin{gathered}
(F g)(\hat{x})=\gamma e^{-i k \hat{x} \cdot z} \\
\gamma=\frac{e^{i \pi / 4}}{\sqrt{8 \pi k}}
\end{gathered}
$$

Our main concern in this paper is to study the far field equation (2.6) for various sampling points $z \in \mathbb{R}^{2}$.

\subsection{The linear sampling method for Dirichlet boundary con- ditions}

In this section we consider the simple case where only Dirichlet data is described on the Lipschitz boundary $\Gamma$, that is $\Gamma_{I}=\emptyset$ in (2.1)-(2.4), and assume that $k^{2}$ is not a Dirichlet eigenvalue for $-\Delta$ in $D$.

The direct scattering problem is then a special case of the exterior Dirichlet boundary value problem

$$
\begin{array}{rlll}
\Delta w+k^{2} w & =0 & \text { in } & \mathbb{R}^{2} \backslash \bar{D} \\
w & =f & \text { on } & \Gamma,
\end{array}
$$

where $w$ satisfies Sommerfeld radiation condition (2.4). This problem has a unique solution $w \in H_{l o c}^{1}\left(\mathbb{R}^{2} \backslash \bar{D}\right)$ provided $f \in H^{1 / 2}(\Gamma)$ (see e.g. [9]), and $w$ has the asymptotic behavior (1.2) at infinity.

Let us define the linear operator $B$ which maps the boundary data $f$ onto the far field pattern $w_{\infty}$ of the radiating solution $w$ of (2.8)-(2.9). Since $w$ depends continuously on the boundary data $f \in H^{1 / 2}(\Gamma)$ the operator $B: H^{1 / 2}(\Gamma) \longrightarrow L^{2}(\Omega)$ is bounded. By superposition we have the following relation

$$
(F g)=-B(\mathcal{H} g),
$$


where $\mathcal{H} g$ is the trace on the boundary $\Gamma$ of the Herglotz wave function $v_{g}$ given by (1.7). Next we define the operator $\mathcal{F}: H^{-1 / 2}(\Gamma) \longrightarrow L^{2}(\Omega)$ by

$$
(\mathcal{F} \phi)(\hat{x})=\int_{\Gamma} \phi(y) e^{-i k \hat{x} \cdot y} d s(y), \quad \hat{x} \in \Omega .
$$

For a given $\phi \in H^{-1 / 2}(\Gamma)$, the function $(\mathcal{F} \phi)(\hat{x})$ is the far field pattern of the radiating solution $\gamma^{-1} \mathcal{S} \phi(x)$ where $\mathcal{S} \phi$ is the single layer potential

$$
\mathcal{S} \phi(x):=\int_{\Gamma} \phi(y) \Phi(x, y) d s(y), \quad x \in \mathbb{R}^{2} \backslash \Gamma,
$$

with $\Phi(x, y)$ defined by (1.4). From the trace theorem we have $\left.\mathcal{S} \phi(x)\right|_{\Gamma}=S \phi(x)$, where $S$ is a boundary integral operator defined by

$$
(S \phi)(x):=\int_{\Gamma} \phi(y) \Phi(x, y) d s(y), \quad x \in \Gamma,
$$

which under our assumption on $k^{2}$ is an isomorphism from $H^{-1 / 2}(\Gamma)$ onto $H^{1 / 2}(\Gamma)$ (see Theorem 7.6 in [9]).

Hence the following relation holds

$$
(\mathcal{F} \phi)=\gamma^{-1} B(S \phi)
$$

Combining (2.10) and (2.14) we obtain the following factorization of the far field operator $F$

$$
(F g)=-\gamma \mathcal{F} S^{-1}(\mathcal{H} g)
$$

and hence the far field equation (2.6) can be writen as

$$
\mathcal{F} S^{-1}(\mathcal{H} g)=-e^{-i k \hat{x} \cdot z}
$$

Theorem 2.1 The operator $\mathcal{F}: H^{-1 / 2}(\Gamma) \longrightarrow L^{2}(\Omega)$ is injective and has dense range provided $k^{2}$ is not a Dirichlet eigenvalue for the negative Laplacian in D.

Proof. Let $\mathcal{F} \phi=0$ and $\phi \in H^{-1 / 2}(\Gamma)$. Then the radiating solution $\mathcal{S} \phi(x)$ has zero far field pattern, whence $\mathcal{S} \phi(x) \equiv 0$ for $x \in \mathbb{R}^{2} \backslash \bar{D}$ (see Theorem 2.13 in [5]). The trace theorem yields $S \phi=0$ almost everywhere on the boundary $\Gamma$, and from the injectivity of the boundary operator $\phi \equiv 0$ in $H^{-1 / 2}(\Gamma)$. Hence $\mathcal{F}$ is injective. The dual (or transpose) operator $\mathcal{F}^{\top}: L^{2}(\Omega) \longrightarrow H^{1 / 2}(\Gamma)$ of $\mathcal{F}$ is given by

$$
\left(\mathcal{F}^{\top} g\right)(y)=\int_{\Omega} g(\hat{x}) e^{-i k \hat{x} \cdot y} d s(\hat{x}) \quad y \in \Gamma .
$$

Let $\mathcal{F}^{\top} g=0$ in $H^{1 / 2}(\Gamma)$. Then

$$
v_{g}(y):=\int_{\Omega} g(\hat{x}) e^{-i k \hat{x} \cdot y} d s(\hat{x})
$$


defines a Herglotz wave function which solves the homogeneous Dirichlet problem in the interior of $\Gamma$. Since $k^{2}$ is not an eigenvalue $v_{g}$ vanishes in $D$, and since $v_{g}$ is analytic in $\mathbb{R}^{2}$ it follows that $v_{g} \equiv 0$ everywhere. Theorem 3.15 of [5] yields $g=0$ on $\Omega$, which means that $\mathcal{F}^{\top}$ is injective.

Now the range $\mathcal{F}$ can be characterize as follows (see e.g. [9], p.23)

$$
\operatorname{kern} \mathcal{F}^{\top}=(\text { range } \mathcal{F})^{a}
$$

where the annihilator (range $\mathcal{F})^{a}$ is a closed subset of $L^{2}(\Omega)$ defined by

$$
(\text { range } \mathcal{F})^{a}=\left\{g \in L^{2}(\Omega):\langle g, \psi\rangle=0 \quad \text { for all } \quad \psi \in \operatorname{range} \mathcal{F}\right\}
$$

with $\langle g, \psi\rangle$ being the duality pairing given by $\int_{\Gamma} g \psi d s$. Therefore from (2.19) and the injectivity of $\mathcal{F}^{\top}$ we have

$$
\left\{g \in L^{2}(\Omega):\langle g, \psi\rangle=0 \quad \text { for all } \quad \psi \in \text { range } \mathcal{F}\right\}=\{0\},
$$

whence the range of $\mathcal{F}$ is dense in $L^{2}(\Omega)$. This ends the proof.

We remark that from (2.14) the range of $\mathcal{F}$ coincides with the range of $B$ since the operator $S: H^{-1 / 2}(\Gamma) \longrightarrow H^{1 / 2}(\Gamma)$ is bijective.

Now let us consider the far field equation in the form (2.16) and assume first that $z \in D$. Since $-e^{-i k \hat{x} \cdot z}$ is in the range of $\mathcal{F}\left(\gamma e^{-i k \hat{x} \cdot z}\right.$ is the far field pattern of the fundamental solution $\Phi(x, z)$ ), it follows that for any $z \in D$ there exists a unique $\phi_{z} \in H^{-1 / 2}(\Gamma)$ such that

$$
\left(\mathcal{F} \phi_{z}\right)(\hat{x})=-e^{-i k \hat{x} \cdot z}
$$

Hence, to solve the far field equation we have to find a $g(\cdot, z) \in L^{2}(\Omega)$ such that $S^{-1} \mathcal{H} g(\cdot, z)=\phi_{z}$ or $\mathcal{H} g(\cdot, z)=S \phi_{z}$. Thus the corresponding Herglotz wave function $v_{g}(\cdot, z)$ solves the Dirichlet problem in $D$ with boundary data $S \phi_{z} \in H^{1 / 2}(\Gamma)$. Unfortunately this cannot always be done. However from the result of Colton and Sleeman [6] (justified for Lipschitz domains in [1]) we can approximate the solution $\mathcal{S} \phi_{z} \in H^{1}(D)$ of the Helmholtz equation by a Herglotz wave function $v_{g}(\cdot, z)$ and therefore by the continuity of the trace operator we can approximate $S \phi_{z} \in H^{1 / 2}(\Gamma)$ by $\mathcal{H} g(\cdot, z):=\left.v_{g}(\cdot, z)\right|_{\Gamma}$. Hence, since $S^{-1}$ is continuous, for every $\epsilon>0$ we can find a $g(\cdot, z) \in L^{2}(\Omega)$ such that

$$
\left\|S^{-1} \mathcal{H} g(\cdot, z)-\phi_{z}\right\|_{H^{-1 / 2}(\Gamma)}<\epsilon
$$


which yields

$$
\left\|\mathcal{F} S^{-1} \mathcal{H} g(\cdot, z)+e^{-i k \hat{x} \cdot z}\right\|_{L^{2}(\Omega)}<c \epsilon,
$$

for some positive constant $c$ or, in other words, $g(\cdot, z) \in L^{2}(\Omega)$ satisfies the far-field inequality

$$
\left\|F g(\cdot, z)-\gamma e^{-i k \hat{x} \cdot z}\right\|_{L^{2}(\Omega)}<c \epsilon .
$$

Furthermore, since $\gamma e^{-i k \hat{x} \cdot z}=\Phi_{\infty}(x, z)$ we have $\left.S \phi_{z} \equiv \Phi(x, z)\right|_{x \in \Gamma \text {. Thus for this }}$ $g(\cdot, z)$ it follows from the boundness of the trace operator and the fact that, for $z \in \Gamma, \Phi(\cdot, z) \notin H^{1}(D)$ that

$$
\lim _{z \rightarrow \Gamma}\left\|v_{g}(\cdot, z)\right\|_{H^{1}(D)}=\infty
$$

and hence

$$
\lim _{z \rightarrow \Gamma}\|g(\cdot, z)\|_{L^{2}(\Omega)}=\infty
$$

Now let us consider $z \in \mathbb{R}^{2} \backslash \bar{D}$. In this case $-e^{-i k \hat{x} \cdot z}$ does not belong to the range of $\mathcal{F}$. But, from Theorem 2.1, by using Tikhonov regularization we can construct a regularized solution of (2.22). In particular, if $\phi_{z}^{\alpha} \in H^{-1 / 2}(\Gamma)$ is the regularized solution of (2.22) corresponding to the regularization parameter $\alpha$ (chosen by a regular regularization strategy e.g. the Morozov discrepancy principle), we have

$$
\left\|\left(\mathcal{F} \phi_{z}^{\alpha}\right)(\hat{x})+e^{-i k \hat{x} \cdot z}\right\|_{L^{2}(\Omega)}<\delta, \quad \delta>0
$$

and

$$
\lim _{\alpha \rightarrow 0}\left\|\phi_{z}^{\alpha}\right\|_{H^{-1 / 2}(\Gamma)}=\infty .
$$

The above considerations for $\phi_{z}$ in the case of $z \in D$ are valid for $\phi_{z}^{\alpha}$ as well. In particular for every $\epsilon^{\prime}>0$ we can find a function $g_{\alpha}(\cdot, z) \in L^{2}(\Omega)$ such that

$$
\left\|S^{-1} \mathcal{H} g_{\alpha}(\cdot, z)-\phi_{z}^{\alpha}\right\|_{H^{-1 / 2}(\Gamma)}<\epsilon^{\prime}
$$

Combining (2.28) and (2.30) we have that $g_{\alpha}(\cdot, z) \in L^{2}(\Omega)$ satisfies

$$
\left\|\mathcal{F} S^{-1} \mathcal{H} g_{\alpha}(\cdot, z)+e^{-i k \hat{x} \cdot z}\right\|_{L^{2}(\Omega)}<\epsilon+\delta
$$

or in other words the far-field inequality

$$
\left\|F g_{\alpha}(\cdot, z)-\gamma e^{-i k \hat{x} \cdot z}\right\|_{L^{2}(\Omega)}<\epsilon+\delta
$$


for $\epsilon>0$ and $\delta>0$ arbitrary small. In addition, (2.29) and (2.30) yield

$$
\lim _{\alpha \rightarrow 0}\left\|\mathcal{H} g_{\alpha}(\cdot, z)\right\|_{H^{1 / 2}(\Gamma)}=\infty
$$

and hence

$$
\lim _{\alpha \rightarrow 0}\left\|g_{\alpha}(\cdot, z)\right\|_{L^{2}(\Gamma)}=\infty
$$

and

$$
\lim _{\alpha \rightarrow 0}\left\|v_{g_{\alpha}}(\cdot, z)\right\|_{H^{1}(D)}=\infty
$$

where $v_{g_{\alpha}}$ is the Herglotz wave function with kernel $g_{\alpha}$.

We summarize these results in the following theorem, noting that for $z \in \mathbb{R}^{2} \backslash \bar{D}$ we have that $\alpha \rightarrow 0$ as $\delta \rightarrow 0$.

Theorem 2.2 Assume that $\Gamma$ is Lipschitz and $k^{2}$ is not a Dirichlet eigenvalue for the negative Laplacian in the interior of $\Gamma$. Then if $F$ is the far field operator corresponding to the scattering problem for Dirichlet boundary conditions, i.e. (2.1)(2.4) with $\Gamma_{I}=\emptyset$, we have that

1) if $z \in D$ then for every $\epsilon>0$ there exists a solution $g^{\epsilon}(\cdot, z) \in L^{2}(\Omega)$ of the inequality

$$
\left\|F g^{\epsilon}(\cdot, z)-\gamma e^{-i k \hat{x} \cdot z}\right\|_{L^{2}(\Omega)}<\epsilon
$$

such that

$$
\lim _{z \rightarrow \Gamma}\left\|g^{\epsilon}(\cdot, z)\right\|_{L^{2}(\Omega)}=\infty \quad \text { and } \quad \lim _{z \rightarrow \Gamma}\left\|v_{g^{\epsilon}}(\cdot, z)\right\|_{H^{1}(D)}=\infty,
$$

where $v_{g^{\epsilon}}$ is the Herglotz wave function with kernel $g^{\epsilon}$, and

2) if $z \in \mathbb{R}^{2} \backslash \bar{D}$ then for every $\epsilon>0$ and $\delta>0$ there exists a solution $g^{\epsilon, \delta}(\cdot, z) \in$ $L^{2}(\Omega)$ of the inequality

$$
\left\|F g^{\epsilon, \delta}(\cdot, z)-\gamma e^{-i k \hat{x} \cdot z}\right\|_{L^{2}(\Omega)}<\epsilon+\delta
$$

such that

$$
\lim _{\delta \rightarrow 0}\left\|g^{\epsilon, \delta}(\cdot, z)\right\|_{L^{2}(\Omega)}=\infty \quad \text { and } \quad \lim _{\delta \rightarrow 0}\left\|v_{g^{\epsilon, \delta}}(\cdot, z)\right\|_{H^{1}(D)}=\infty
$$

where $v_{g^{\epsilon, \delta}}$ is the Herglotz wave function with kernel $g^{\epsilon, \delta}$.

In the Introduction we claimed that the above analysis provides a mathematical explanation for the numerical behaviour exhibited in the implementation of the first 
version of the linear sampling method. A legitimate criticism of this statement is that we do not know how well the regularized solution of

$$
F g=\Phi_{\infty}
$$

approximates the solution $g$ of

$$
F g=\Phi_{\infty}^{\epsilon, \delta}
$$

where $\Phi_{\infty}^{\epsilon, \delta}$ is the approximation of $\Phi_{\infty}$ due to the approximation of the far field pattern $\Phi_{\infty}$ (measured by $\delta$ ) and the approximation of the solution of the Helmholtz equation by a Herglotz wave function (measured by $\epsilon$ ). In particular since in general a solution of (2.36) does not exist for $z$ either in $D$ or $\mathbb{R}^{2} \backslash \bar{D}$ it makes no sense to let $\epsilon$ and $\delta$ tend to zero, i.e. $\epsilon$ and $\delta$ are fixed parameters. However the same criticism applies in practice to any regularization scheme since noise is not a variable but rather a fixed parameter. In particular even if an exact solution to (2.36) did exist in a noise free environment (i.e. $u_{\infty}$ is free of noise) in practice the kernel is noisy and one has no idea if the noise is small enough so that the regularized solution of the equation with noisy data is in fact a good approximation to the solution of (2.36) with noise free data. The only statement that can be made is what happens if the noise tends to zero. However, since the noise is fixed and nonzero, in either case the analysis leads to the same conclusion: there is a "nearby" equation ( $F$ with noisy kernel and, in the case of (2.37) also with inexact right hand side) whose solution behaves in a known way and if this "nearby" equation is "close enough" to (2.36) (with $u_{\infty}$ free of noise) then one expects the regularized solution to behave like the known solution. In particular, since error estimates are not available for the dependency of the regularized solution on the noise level, the remark of Lanczos is valid: "a lack of information cannot be remedied by any mathematical trickery". Nevertheless, an explanation such as that given above or the arguments of [7], [8] are valuable since they provide an explanation of the observed numerical behaviour of the regularized solution and thus an understanding why the linear sampling method works.

\subsection{The linear sampling method for mixed boundary condi- tions}

In this section we show that the argument of the previous analysis can be easily applied to the more general case of the obstacle scattering problems involving partially coated obstacles or multiple scattering objects with different boundary conditions. 
To our knowledge the first version of the linear sampling method considered here is the only numerically viable method for solving inverse scattering problems in the case of mixed boundary conditions (see [1]). Here we give a mathematical justification of the method.

We consider the Lipschitz boundary $\Gamma$ dissected $\Gamma=\Gamma_{D} \cup \Pi \cup \Gamma_{I}$ as described in Section 2.1, and furthermore assume that $\Gamma_{I} \neq \emptyset$. The direct obstacle scattering problem (2.1)-(2.4) is a special case of the exterior mixed boundary value problem

$$
\begin{array}{rlrlrl}
\Delta w+k^{2} w & =0 & \text { in } & \mathbb{R}^{2} \backslash \bar{D}, \\
w=f & \text { on } & & \Gamma_{D}, \\
\frac{\partial w}{\partial \nu}+i \lambda k w=h & \text { on } & \Gamma_{I},
\end{array}
$$

where $\lambda \geq 0$ is a constant, and $w$ satisfies Sommerfeld radiation condition (2.4). If $f \in H^{1 / 2}\left(\Gamma_{D}\right)$ and $h \in H^{-1 / 2}\left(\Gamma_{I}\right)$ then the exterior mixed boundary value problem has a unique solution $w \in H_{l o c}^{1}\left(\mathbb{R}^{2} \backslash \bar{D}\right)[1]$, and $w$ has the asymptotic behavior (1.2) at infinity with far field pattern $w_{\infty}$. We recall that for $\Gamma_{0} \subseteq \Gamma$

$$
\begin{aligned}
& H^{1 / 2}\left(\Gamma_{0}\right):=\left\{\left.u\right|_{\Gamma_{0}}: u \in H^{1 / 2}(\Gamma)\right\} \\
& \tilde{H}^{1 / 2}\left(\Gamma_{0}\right):=\left\{u \in H^{1 / 2}(\Gamma): \operatorname{supp} u \subseteq \bar{\Gamma}_{0}\right\},
\end{aligned}
$$

and moreover $H^{-1 / 2}\left(\Gamma_{0}\right):=\left(\tilde{H}^{1 / 2}\left(\Gamma_{0}\right)\right)^{\prime}$ and $\tilde{H}^{-1 / 2}\left(\Gamma_{0}\right):=\left(H^{1 / 2}\left(\Gamma_{0}\right)\right)^{\prime}$.

Let $B$ be the bounded operator from $H^{1 / 2}\left(\Gamma_{D}\right) \times H^{-1 / 2}\left(\Gamma_{I}\right)$ onto $L^{2}(\Omega)$ which maps the boundary data $(f, h)$ to the far field pattern $w_{\infty}$. Thus, the far field operator (2.5) corresponding to this case can be written

$$
(F g)=-B(\mathcal{H} g)
$$

where now the boundary trace $\mathcal{H} g$ of the Herglotz wave function is given by

$$
\mathcal{H} g(x):=\left\{\begin{array}{cc}
\int_{\Omega} g(d) e^{i k x \cdot d} d s(d) & x \in \Gamma_{D} \\
\frac{\partial}{\partial \nu_{x}} \int_{\Omega} g(d) e^{i k x \cdot d} d s(d)+i k \lambda \int_{\Omega} g(d) e^{i k x \cdot d} d s(d) & x \in \Gamma_{I}
\end{array}\right.
$$

We define the operator $\mathcal{F}: \tilde{H}^{-1 / 2}\left(\Gamma_{D}\right) \times \tilde{H}^{1 / 2}\left(\Gamma_{I}\right) \longrightarrow L^{2}(\Omega)$ by

$$
\begin{aligned}
\mathcal{F}\left(\phi_{D}, \phi_{I}\right)(\hat{x}) & =\int_{\Gamma_{D}} \phi_{D}(y) e^{-i k \hat{x} \cdot y} d s(y) \quad \hat{x} \in \Omega \\
& +\int_{\Gamma_{I}} \phi_{I}(y) \frac{\partial}{\partial \nu_{y}} e^{-i k \hat{x} \cdot y} d s(y)+i k \lambda \int_{\Gamma_{I}} \phi_{I}(y) e^{-i k \hat{x} \cdot y} d s(y),
\end{aligned}
$$

and observe that for a given pair $\left(\phi_{D}, \phi_{I}\right) \in \tilde{H}^{-1 / 2}\left(\Gamma_{D}\right) \times \tilde{H}^{1 / 2}\left(\Gamma_{I}\right)$, the function $\mathcal{F}\left(\phi_{D}, \phi_{I}\right)(\hat{x})$ is the far field pattern of the radiating solution $\gamma^{-1} \mathcal{P}\left(\phi_{D}, \phi_{I}\right)(x)$ with

$$
\mathcal{P}\left(\phi_{D}, \phi_{I}\right):=\mathcal{S} \tilde{\phi}_{D}+\mathcal{D} \tilde{\phi}_{I}+i k \lambda \mathcal{S} \tilde{\phi}_{I}
$$


where $\tilde{\phi}_{D} \in H^{-1 / 2}(\Gamma)$ and $\tilde{\phi}_{I} \in H^{1 / 2}(\Gamma)$ are the extensions by zero of $\phi_{D}$ with support in $\bar{\Gamma}_{D}$ and $\phi_{I}$ with support in $\bar{\Gamma}_{I}$, respectively. Here $\mathcal{S} \phi$ denotes the single layer potential (2.12) and $\mathcal{D} \phi$ double layer potential defined by

$$
\mathcal{D} \phi(x):=\int_{\Gamma} \phi(y) \frac{\partial}{\partial \nu_{y}} \Phi(x, y) d s(y), \quad x \in \mathbb{R}^{2} \backslash \Gamma .
$$

Since $\left.\phi_{D}\right|_{\Gamma_{I}} \equiv 0$ and $\left.\phi_{I}\right|_{\Gamma_{D}} \equiv 0$, the trace theorem and jump relations of the singleand double- layer potential on the boundary $\Gamma$ yield

$$
\left(\begin{array}{r}
\left.\mathcal{P}\left(\phi_{D}, \phi_{I}\right)\right|_{\Gamma_{D}} \\
\left.\left(\frac{\partial}{\partial \nu}+i k \lambda\right) \mathcal{P}\left(\phi_{D}, \phi_{I}\right)\right|_{\Gamma_{I}}
\end{array}\right)=M\left(\begin{array}{c}
\phi_{D} \\
\phi_{I}
\end{array}\right)
$$

where the operator $M$ is given by

$$
M=\left(\begin{array}{cc}
S_{D D}, & K_{D I}+i k \lambda S_{D I} \\
K_{I D}^{\prime}+i k \lambda S_{I D}, & -k^{2} \lambda^{2} S_{I I}+i k \lambda\left(K_{I I}^{\prime}+K_{I I}\right)+T_{I I}
\end{array}\right) .
$$

Here $S, K, K^{\prime}, T$ denote the four basic boundary integral operators defined by

$$
\begin{array}{rlrl}
S \phi(x):=2 \int_{\Gamma} \phi(y) \Phi(x, y) d s_{y} & K \phi(x):=2 \int_{\Gamma} \phi(y) \frac{\partial}{\partial \nu_{y}} \Phi(x, y) d s_{y} \\
K^{\prime} \phi(x):=2 \int_{\Gamma} \phi(y) \frac{\partial}{\partial \nu_{x}} \Phi(x, y) d s_{y} & T \phi(x):=2 \frac{\partial}{\partial \nu_{x}} \int_{\Gamma} \psi(y) \frac{\partial}{\partial \nu_{y}} \Phi(x, y) d s_{y}
\end{array}
$$

the operator $S_{I D}$ is the operator $S$ applied to a function $\phi$ with supp $\phi \subseteq \bar{\Gamma}_{D}$ and evaluated on $\Gamma_{I}$, with analogous definitions for $S_{D D}, S_{D I}, S_{I I}, K_{D I}, K_{I D}^{\prime}, K_{I I}, K_{I I}^{\prime}$ and $T_{I I}$. In [1] it is proved that provided $\lambda>0$ and $\Gamma_{I} \neq \emptyset$ the operator

$$
A=M \cdot\left(\begin{array}{cc}
I & 0 \\
0 & -I
\end{array}\right): \tilde{H}^{-1 / 2}\left(\Gamma_{D}\right) \times \tilde{H}^{1 / 2}\left(\Gamma_{I}\right) \longrightarrow H^{1 / 2}\left(\Gamma_{D}\right) \times H^{-1 / 2}\left(\Gamma_{I}\right),
$$

with $I$ the identity operator, is bijective. Hence, if we define $H:=\tilde{H}^{-1 / 2}\left(\Gamma_{D}\right) \times$ $\tilde{H}^{1 / 2}\left(\Gamma_{I}\right)$, and its dual $H^{*}:=H^{1 / 2}\left(\Gamma_{D}\right) \times H^{-1 / 2}\left(\Gamma_{I}\right)$ then $M$ is an isomorphism from $H$ onto $H^{*}$.

Obviously the following relation holds

$$
\mathcal{F}\left(\phi_{D}, \phi_{I}\right)=-\gamma B M\left(\phi_{D}, \phi_{I}\right),
$$

and hence the far field equation can be written as

$$
\mathcal{F} M^{-1}(\mathcal{H} g)=-e^{-i k \hat{x} \cdot z}
$$


Theorem 2.3 Assume that $\lambda>0$ and $\Gamma_{I} \neq \emptyset$. Then the operator $\mathcal{F}: H \longrightarrow L^{2}(\Omega)$ defined by (2.44) is injective and has dense range.

Proof. The proof of the theorem proceeds in exactly the same way as in Theorem 2.1. We note that in this case the dual operator $\mathcal{F}^{\top}: L^{2}(\Omega) \longrightarrow H^{*}$ is given by

$$
\left(\mathcal{F}^{\top} g\right)(y):=\left\{\begin{array}{cc}
\int_{\Omega} g(\hat{x}) e^{-i k \hat{x} \cdot y} d s(\hat{x}) & y \in \Gamma_{D} \\
\frac{\partial}{\partial \nu_{x}} \int_{\Omega} g(d) e^{-i k \hat{x} \cdot y} d s(\hat{x})+i k \lambda \int_{\Omega} g(\hat{x}) e^{-i k \hat{x} \cdot y} d s(\hat{x}) & y \in \Gamma_{I} .
\end{array}\right.
$$

Moreover in the proof of this theorem the bijective operator $M$ given by (2.48) plays the role of the operator $S$ in the Theorem 2.1.

We are now ready to analyse the far field equation written in the form (2.50). We proceed for $\mathcal{F}$ given by (2.44) and $\mathcal{H}$ given by (2.43) in the same way as in Section 2.2. Note that the role of the weak solution $\mathcal{S} \phi_{z} \in H^{1}(D)$ and the boundary operator $S$ is now replaced by the weak solution $\mathcal{P}\left(\phi_{D}, \phi_{I}\right) \in H^{1}(D)$ and the boundary operator $M$, respectively. Hence we conclude that Theorem 2.2 is also valid for the far field operator $F$ corresponding to the scattering problem (2.1)-(2.4) provided that $\lambda>0$ and $\Gamma_{I} \neq \emptyset$ :

Theorem 2.4 Assume that $\Gamma$ is Lipschitz having a Lipschitz dissection $\Gamma=\Gamma_{D} \cup$ $\Pi \cup \Gamma_{I}$ with $\Gamma_{I} \neq \emptyset$, and $\lambda>0$. Then if $F$ is the far field operator corresponding to (2.1)-(2.4) we have that

1) if $z \in D$ then for every $\epsilon>0$ there exists a solution $g^{\epsilon}(\cdot, z) \in L^{2}(\Omega)$ of the inequality

$$
\left\|F g^{\epsilon}(\cdot, z)-\gamma e^{-i k \hat{x} \cdot z}\right\|_{L^{2}(\Omega)}<\epsilon
$$

such that

$$
\lim _{z \rightarrow \Gamma}\left\|g^{\epsilon}(\cdot, z)\right\|_{L^{2}(\Omega)}=\infty \quad \text { and } \quad \lim _{z \rightarrow \Gamma}\left\|v_{g^{\epsilon}}(\cdot, z)\right\|_{H^{1}(D)}=\infty,
$$

where $v_{g^{\epsilon}}$ is the Herglotz wave function with kernel $g^{\epsilon}$, and

2) if $z \in \mathbb{R}^{2} \backslash \bar{D}$ then for every $\epsilon>0$ and $\delta>0$ there exists a solution $g^{\epsilon, \delta}(\cdot, z) \in$ $L^{2}(\Omega)$ of the inequality

$$
\left\|F g^{\epsilon, \delta}(\cdot, z)-\gamma e^{-i k \hat{x} \cdot z}\right\|_{L^{2}(\Omega)}<\epsilon+\delta
$$

such that

$$
\lim _{\delta \rightarrow 0}\left\|g^{\epsilon, \delta}(\cdot, z)\right\|_{L^{2}(\Omega)}=\infty \quad \text { and } \quad \lim _{\delta \rightarrow 0}\left\|v_{g^{\epsilon, \delta}}(\cdot, z)\right\|_{H^{1}(D)}=\infty
$$

where $v_{g^{\epsilon, \delta}}$ is the Herglotz wave function with kernel $g^{\epsilon, \delta}$. 
We remark that if $\lambda=0$, i.e. the Neumann boundary condition is assumed on $\Gamma_{I}$, the same conclusion remains valid provided $k^{2}$ is not an eigenvalue for the interior homogeneous mixed boundary value problem in $D$.

In the particular case of the impedance problem, i.e. $\Gamma_{D}=\emptyset, \lambda>0$, we obtain the same result as in Theorem 2.4 by an appropriate modification of the previous analysis.

\section{The linear sampling method for the inverse medium problem}

We now turn our attention to the scattering of a plane wave by a penetrable inhomogeneous medium of compact support. In particular, consider the direct scattering problem of finding $u \in C^{2}\left(\mathbb{R}^{2} \backslash \Gamma\right) \cup C^{1}\left(\mathbb{R}^{2}\right)$ such that

$$
\begin{aligned}
& \Delta u+k^{2} n(x) u=0 \quad \text { in } \quad \mathbb{R}^{2} \backslash \Gamma, \\
& u(x)=e^{i k x \cdot d}+u^{s}(x), \\
& \lim _{r \rightarrow \infty}\left(\frac{\partial u^{s}}{\partial r}-i k u^{s}\right)=0 .
\end{aligned}
$$

The index of refraction $n$ is assumed to be piecewise continuously differentiable such that $m:=1-n$ has compact support $\bar{D} \subset \mathbb{R}^{2}$ where the complement of $\bar{D}$ is connected and $\bar{D}$ has smooth boundary $\Gamma$ with unit outward normal $\nu$. More specifically, we assume that $n$ is smooth except for a jump discontinuity across $\Gamma$. We will further restrict ourselves to the case when $\operatorname{Im} n(x) \geq c>0$ for $x \in D$ and $c$ a constant (absorbing medium). However, if we have that $\operatorname{Im} n(x)=0$ for $x \in D$ (nonabsorbing medium), then the analysis that follows remains valid if we assume that $k$ is not a transmission eigenvalue (c.f. [5] for the definition of a transmission eigenvalue).

Under these assumptions it is known [5] that there exists a unique solution $u$ to (3.1)-(3.3) and $u$ has the asymptotic behavior (1.2) at infinity with the far field pattern $u_{\infty}$ given by

$$
u_{\infty}(\hat{x} ; d)=-\gamma \int_{D} e^{-i k \hat{x} \cdot y} m(y) u(y) d y, \quad \hat{x} \in \Omega
$$

The scattering problem (3.1)-(3.3) can be written as the integral equation

$$
e^{i k x \cdot d}=u(x, d)+k^{2} T u(\cdot, d)(x),
$$


with the operator $T: L^{2}(D) \longrightarrow L^{2}(D)$ defined by

$$
(T \varphi)(x):=\int_{D} \Phi(x, y) m(y) \varphi(y) d y \quad \text { for } \quad x \in D
$$

where $\Phi(x, y)$ is given by (1.4). It is known [5] that the operator $I+k^{2} T$ is an isomorphism from $L^{2}(D)$ onto itself.

The inverse medium scattering problem we discuss in this section is to determine the support $D$ of $m=1-n$ from a knowledge of $u_{\infty}(\hat{x}, d)$ for $\hat{x}$ and $d$ on the unit circle $\Omega$ and fixed wave number $k$. We use the linear sampling method to solve the inverse medium problem. In other words, our aim is to show again in this case that there exists an approximate solution $g(\cdot, z)$ of the far field equation (1.3) such that $\|g(\cdot, z)\|_{L^{2}(\Omega)}$ and $\left\|v_{g}(\cdot, z)\right\|_{L^{2}(D)}$, become unbounded as $z$ tends to $\Gamma$ and remain so for $z \in \mathbb{R}^{2} \backslash D$.

If $v_{g}$ is a Herglotz wave function with kernel $g$, than from (3.4) the far field equation (2.5) takes the form

$$
-\gamma \int_{D} e^{-i k \hat{x} \cdot y} m(y)\left(I+k^{2} T\right)^{-1} v_{g}(y) d y=\gamma e^{-i k \hat{x} \cdot z}, \quad z \in \mathbb{R}^{2},
$$

where $\gamma$ is given by (2.7). Denote by $\bar{H}$ the closure of the set of Herglotz wave functions in $L^{2}(D)$ and define the operator $\mathcal{F}: L^{2}(D) \longrightarrow L^{2}(\Omega)$ by

$$
(\mathcal{F} \varphi)(\hat{x}):=\gamma \int_{D} e^{-i k \hat{x} \cdot y} m(y) \varphi(y) d y \quad \hat{x} \in \Omega
$$

Hence, in terms of the operator $\mathcal{F}$ the far field equation (3.7) can be written as

$$
\mathcal{F}\left(I+k^{2} T\right)^{-1} v_{g}=-e^{-i k \hat{x} \cdot z} .
$$

We note that $\mathcal{F}\left(I+k^{2} T\right)^{-1}$ is clearly a bounded operator from $L^{2}(D)$ onto itself.

For the following analysis we recall the interior transmission problem of finding a pair of functions $v, w$ such that

$$
\Delta w+k^{2} n(x) w=0, \quad \Delta v+k^{2} v=0 \quad \text { in } \quad D,
$$

and $v, w$ satisfy the transmission conditions

$$
w-v=\Phi(\cdot, z), \quad \frac{\partial w}{\partial \nu}-\frac{\partial w}{\partial \nu}=\frac{\partial}{\partial \nu} \Phi(\cdot, z) \quad \text { on } \quad \Gamma .
$$

For $z \in D$, this problem has a unique weak solution $v \in \bar{H}$ and $w \in L^{2}(D)$, (for the definition of a weak solution and the proof of this statement see [3] or Theorem 10.24, Theorem 10.25 in [5]). 
Theorem 3.1 The operator $\mathcal{F}\left(I+k^{2} T\right)^{-1}: \bar{H} \subset L^{2}(D) \longrightarrow L^{2}(\Omega)$ is injective and has dense range.

Proof. Let $\mathcal{F}\left(I+k^{2} T\right)^{-1} v=0$ for a $v \in \bar{H}$ and set $w=\left(I+k^{2} T\right)^{-1} v$. Then the pair $v \in \bar{H}$ and $w \in L^{2}(D)$ is a solution of the homogenous interior transmission problem, and by uniqueness $v \equiv 0$. Hence $\mathcal{F}\left(I+k^{2} T\right)^{-1}$ is injective.

Now, let us suppose that there exists a $g \in L^{2}(\Omega)$ such that for all $v \in \bar{H}$ we have

$$
\left(\mathcal{F}\left(I+k^{2} T\right)^{-1} v, g\right)=0
$$

Then the far field patterns corresponding to all Herglotz wave functions as incident fields are orthogonal to $g$. But far field patterns corresponding to the incident fields $e^{i k x \cdot d}, d \in \Omega$, are complete in $L^{2}(\Omega)$ (Theorem 8.12 in [5]) whence far field patterns corresponding to all Herglotz functions are complete in $L^{2}(\Omega)$. Hence $g \equiv 0$, and therefore the range $\mathcal{F}\left(I+k^{2} T\right)^{-1}(\bar{H})$ is dense in $L^{2}(\Omega)$. This ends the proof.

Now let us consider the far field equation (3.9). If $z \in D$, we have that $e^{-i k \hat{x} \cdot z}$ is in the range of $\mathcal{F}\left(I+k^{2} T\right)^{-1}$. Then, let $v(\cdot, z) \in \bar{H}$ and $w(\cdot, z)=\left(I+k^{2} T\right)^{-1} v(\cdot, z) \in L^{2}(D)$ be the unique weak solution of the interior transmission problem (3.10)-(3.11). In this case it can be shown that (see Theorem 10.26 in [5]) for every $\epsilon>0$ the kernel $g(\cdot, z) \in L^{2}(\Omega)$ of the Herglotz wave function $v_{g}(\cdot, z)$ which approximates $v(\cdot, z) \in \bar{H}$, i.e $\left\|v(\cdot, z)-v_{g}(\cdot, z)\right\|_{L^{2}(\Omega)}<\epsilon^{\prime}$ for sufficiently small $\epsilon^{\prime}>0$, solves the inequality

$$
\left\|\mathcal{F}\left(I+k^{2} T\right)^{-1} v_{g}(\cdot, z)+e^{-i k \hat{x} \cdot z}\right\|_{L^{2}(\Omega)}<\epsilon, \quad z \in D,
$$

and moreover satisfies

$$
\lim _{z \rightarrow \Gamma}\|g(\cdot, z)\|_{L^{2}(\Gamma)}=\infty \quad \text { and } \quad \lim _{z \rightarrow \Gamma}\left\|v_{g}(\cdot, z)\right\|_{L^{2}(D)}=\infty
$$

If $z \in \mathbb{R}^{2} \backslash \bar{D}$, then $e^{-i k \hat{x} \cdot z}$ is not in the range of $\mathcal{F}\left(I+k^{2} T\right)^{-1}$. But, from Theorem 3.1, by using Tikhonov regularization we can construct a regularized solution $v^{\alpha}(\cdot, z) \in$ $\bar{H}$ depending on the regularization parameter $\alpha$ such that

$$
\left\|\mathcal{F}\left(I+k^{2} T\right)^{-1} v^{\alpha}(\cdot, z)+e^{-i k \hat{x} \cdot z}\right\|_{L^{2}(\Omega)}<\delta, \quad z \in \mathbb{R}^{2} \backslash \bar{D}
$$

for an arbitrary small $\delta>0$, and

$$
\lim _{\alpha \rightarrow 0}\left\|v^{\alpha}(\cdot, z)\right\|_{L^{2}(D)}=\infty .
$$

By the definition of the space $\bar{H}$ we can approximate $v^{\alpha}(\cdot, z) \in \bar{H}$ by a Herglotz wave function $v_{g_{\alpha}}(\cdot, z)$ with kernel $g_{\alpha}(\cdot, z)$, i.e., for every $\epsilon^{\prime}>0$ there exists $g_{\alpha}(\cdot, z) \in L^{2}(\Omega)$ such that

$$
\left\|v^{\alpha}(\cdot, z)-v_{g_{\alpha}}(\cdot, z)\right\|_{L^{2}(D)}<\epsilon^{\prime}
$$


Then by continuity of $\mathcal{F}\left(I+k^{2} T\right)^{-1}$ we have that

$$
\left\|\mathcal{F}\left(I+k^{2} T\right)^{-1} v^{\alpha}(\cdot, z)-\mathcal{F}\left(I+k^{2} T\right)^{-1} v_{g_{\alpha}}(\cdot, z)\right\|_{L^{2}(D)}<\bar{c} \epsilon^{\prime}
$$

for some positive constant $\bar{c}$. If we now combine the inequalities (3.15)-(3.18) we get

$$
\left\|\mathcal{F}\left(I+k^{2} T\right)^{-1} v_{g_{\alpha}}(\cdot, z)+e^{-i k \hat{x} \cdot z}\right\|_{L^{2}(D)}<\epsilon+\delta, \quad z \in \mathbb{R}^{2} \backslash \bar{D},
$$

with $\epsilon=\bar{c} \epsilon^{\prime}$. Moreover, both inequalities (3.16)-(3.17) imply that

$$
\lim _{\alpha \rightarrow 0}\left\|v_{g_{\alpha}}(\cdot, z)\right\|_{L^{2}(D)}=\infty
$$

whence

$$
\lim _{\alpha \rightarrow 0}\left\|g_{\alpha}(\cdot, z)\right\|_{L^{2}(\Omega)}=\infty .
$$

Noting that $\alpha \rightarrow 0$ as $\delta \rightarrow 0$, we have proved the following theorem for the far field operator $F$ corresponding to the scattering problem (3.1)-(3.3) provided $\operatorname{Im} n(x) \geq$ $c>0$ for $x \in D$ :

Theorem 3.2 Assume that $\Gamma$ is smooth and $\operatorname{Imn}(x) \geq c>0$ for $x \in D$. Then if $F$ is the far field operator corresponding to (3.1)-(3.3) we have that

1) if $z \in D$ then for every $\epsilon>0$ there exists a solution $g^{\epsilon}(\cdot, z) \in L^{2}(\Omega)$ of the inequality

$$
\left\|F g^{\epsilon}(\cdot, z)-\gamma e^{-i k \hat{x} \cdot z}\right\|_{L^{2}(\Omega)}<\epsilon
$$

such that

$$
\lim _{z \rightarrow \Gamma}\left\|g^{\epsilon}(\cdot, z)\right\|_{L^{2}(\Omega)}=\infty \quad \text { and } \quad \lim _{z \rightarrow \Gamma}\left\|v_{g^{\epsilon}}(\cdot, z)\right\|_{L^{2}(D)}=\infty
$$

where $v_{g^{\epsilon}}$ is the Herglotz wave function with kernel $g^{\epsilon}$, and

2) if $z \in \mathbb{R}^{2} \backslash \bar{D}$ then for every $\epsilon>0$ and $\delta>0$ there exists a solution $g^{\epsilon, \delta}(\cdot, z) \in$ $L^{2}(\Omega)$ of the inequality

$$
\left\|F g^{\epsilon, \delta}(\cdot, z)-\gamma e^{-i k \hat{x} \cdot z}\right\|_{L^{2}(\Omega)}<\epsilon+\delta
$$

such that

$$
\lim _{\delta \rightarrow 0}\left\|g^{\epsilon, \delta}(\cdot, z)\right\|_{L^{2}(\Omega)}=\infty \quad \text { and } \quad \lim _{\delta \rightarrow 0}\left\|v_{g^{\epsilon, \delta}}(\cdot, z)\right\|_{L^{2}(D)}=\infty
$$

where $v_{g^{\epsilon, \delta}}$ is the Herglotz wave function with kernel $g^{\epsilon, \delta}$. 
We end by noting that the assumption on the smoothness of the refraction index $n$ and of the boundary $\Gamma$ can be weakened. One can consider for example that $n$ is a complex valued Lipschitz function exept for a jump discontinuity across a Lipschitz curve $\Gamma$ and the above analysis remains valid.

\section{Acknowledgments}

The research of the second author, was supported in part by a grant from the Air Force Office of Scientific Research. The first author was supported in part by NSF Grant 9631287 at the University of Delaware.

\section{References}

[1] Cakoni, F., Colton, D. and Monk, P., The direct and inverse scattering problems for partially coated obstacles, (to appear).

[2] Colton, D., Coyle J. and Monk, P., Recent developments in inverse acoustic scattering theory, SIAM Review, (2000) 42, No. 3, pp. 369-414.

[3] Colton, D., Piana, M. and Potthast, R., A simple method using Morozov's discrepancy principle for solving inverse scattering problems, Inverse Problems, (1997) 13, pp. 1477-1493.

[4] Colton, D. and Kirsch, A., A simple method for solving inverse scattering problems in the resonance region, Inverse Problems, (1996) 12, pp. 383-393.

[5] Colton, D. and Kress, R., Inverse Acoustic and Electromagnetic Scattering Theory, 2nd edn, (1998) Springer Verlag.

[6] Colton, D. and Sleeman, B., An approximation property of importance in inverse scattering theory, Proc. Edinburgh Math. Soc., to appear.

[7] Kirsch, A., Characterization of the shape of a scattering obstacle using the spectral data of the far-field operator, Inverse Problems, (1998) 14, pp. 14891512 .

[8] Kirsch, A., Factorization of the far field operator for the inhomogeneous medium case and an application in inverse scattering theory, Inverse Problems, (1999) 15, pp. 413-429. 
[9] McLean, W., Strongly Elliptic Systems and Boundary Integral Equations, (2000) Cambridge University Press.

[10] Norris, A., A direct inverse scattering method for imaging obstacles with unknown surface conditions, IMA Jour. Applied Math. (1998) 61, pp. 267-290.

[11] Xu Y., Mawata C. and Lin, W., Generalized dual space indicator method for underwater imaging, Inverse Problems, (2000) 16, pp. 1761-1776.

[12] You, Y.X., Miao G.P. and Liu, Y.Z., A fast method for acoustic imaging of multiple three-dimensional objects, J. Acoust. Soc. Amer. (2000) 108, no. 2, pp. 31-37. 